\title{
Medical Quality-of-Service Optimization in Wireless Telemedicine System Using Optimal Smoothing Algorithm
}

\author{
Sodhro Ali Hassan, Ye Li* \\ Shenzhen Institutes of the Advanced Technologies, Chinese Academy of Sciences, Shenzhen, China \\ Email: ali.hassan@siat.ac.cn, ${ }^{*}$ ye.li@siat.ac.cn
}

Received December 17, 2012; revised January 20, 2013; accepted January 26, 2013

\begin{abstract}
In this paper we consider the transmission of stored video from a server to a client for medical applications such as, Tele-monitoring, to optimize medical quality of service (m-QoS) and to examine how the client buffer space can be used efficiently and effectively towards reducing the rate variability of the compressed variable bit rate (VBR) video. Three basic results are presented. First, we show how to obtain the greatest possible reduction in rate variability when sending stored video to client with a given buffer size. Second, how to reduce high peak data rate of compressed VBR video when a patient is moving/walking very fast in hospital. Third, we evaluate the impact of optimal smoothing algorithm on the network parameters such as, peak-to-mean ratio, standard deviation, delay, jitter, average delay and average jitter to optimize the m-QoS. To resolve these all problems we used optimal smoothing algorithm and show its performance over a set of long MPEG-4 encoded video traces. Simulation results show that $\mathrm{m}-\mathrm{QoS}$ is optimized by minimizing network metrics.
\end{abstract}

Keywords: Wireless Telemedicine System; Medical QoS; Optimal Smoothing Algorithm; Mobile Health

\section{Introduction}

Wireless Telemedicine is a way of distributing medical expertise and services in remote area hospitals, there have been greater demands to replace wires with wireless technologies. This replacement not only reduces the deployment cost and time, but also provides patient's increased mobility and comfort by replacing them from wires. The use of wireless technologies enables healthcare personnel to remotely monitor the patient's conditions. Also we can prevent the infection due to wire contact by using wireless technologies. For successful deployment of wireless technologies in healthcare applications, the main challenge is how to guarantee the required quality of service (QoS) level for medical applications when using wireless connection, i.e., medical quality of service (m-QoS) [1]. As mobile healthcare (mhealth) is a new paradigm that brings together the evolution of emerging wireless communications and network technologies with concept of connected healthcare anytime, anywhere to anyone [2].

Until now, there has not been much work on m-QoS using optimal smoothing algorithm. J. D. Salehi. et al. [3] propose an optimal smoothing algorithm for reducing

${ }^{*}$ Corresponding author. rate variability in wireless VBR stored video but ignored $\mathrm{m}$-QoS optimization and trade-off between buffer sizes, peak-to-mean ratio, standard deviation, delay, jitter, average delay and average jitter through optimal smoothing algorithm.

Ye Li et al. [4] design optimal smoothing algorithm for the energy efficient video transmission over wireless link but not focused on the m-QoS optimization and relationship between buffer size, delay, jitter, average delay and average jitter. Ali Alnejad et al. [5] mapped m-health QoS parameters with WiMAX QoS classes. Yishay Mansour et al. [6] present optimal smoothing schedule for real-time streams. Malindi P. [7] present QoS for IPbased Telemedicine system. Robert S. H. Istepanian, et al. [8] develop rate control techniques for $\mathrm{m}-\mathrm{QoS}$ provisioning. Wu-Chi, et al. [9] propose bandwidth smoothing algorithm to reduce burstiness resources. Taceittin, et al. [10] present lossless smoothing algorithm for the delay \& buffer constraint. Ray-I Chang, et al. [11] design linear CTS algorithm for the jitter-free playback. Szymanski, et al. [12] examine QoS in mission-critical telerobotic. B. Woodward et al. [13] design telemedicine system using mobile telephone. [14,15] Presented online smoothing algorithm. Zilei Wang, et al. [16] develop fast optimal smoothing algorithm for stored video. [17-19] discussed 
QoS requirements for general and medical applications respectively.

One of the key limitations of existing works is that they have focused on QoS provisioning in different areas such as broadband networks, cellular networks, robotics etc by using distinct techniques and algorithms but they have not focused on the optimization of m-QoS through optimal smoothing algorithm for medical applications such as Tele-monitoring.

The main contribution of this research is that we first used optimal smoothing algorithm to reduce rate variability and high peak data rates of VBR compressed stored medical video as shown in Figure 1, second established trade-off between client buffer sizes, peak-to-mean ratio, standard deviation, delay, jitter, average delay and average jitter to optimize m-QoS as shown in Table $\mathbf{1}$.

The remainder of the paper is organized as follows. Section 2 introduces wireless telemedicine system. Section 3 presents general QoS. Section 4 describes medical QoS. Section 5 presents difference between general video and medical video. Section 6 describes optimal smoothing of stored/offline medical video, problem statement and optimal smoothing algorithm in detail. Simulation results are presented in Section 7. Section 8 concludes paper.

\section{Wireless Telemedicine System}

Wireless telemedicine is the delivery of health care and sharing of medical knowledge over a distance using Information Communication Technology (ICT) as a communication platform. It aims at providing medical services anywhere at any time. Though any communication

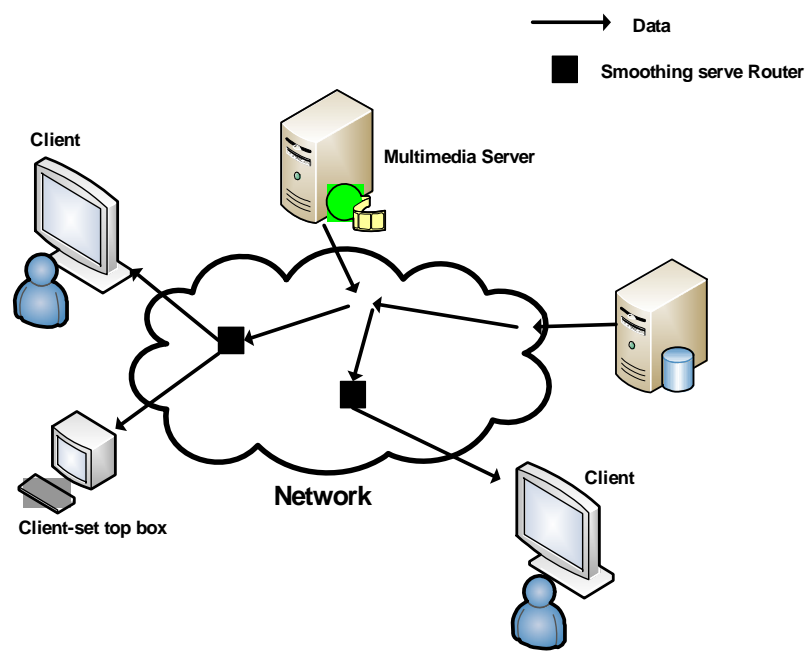

Figure 1. Stored video stream originates from a multimedia server or a video-on-demand system and travels through the network to one or more clients including workstations and set-top boxes. Smoothing occurs at the video source and/or at servers inside the network [3]. system can be used in telemedicine, rapid development in computer technology and easiness to purchase has led to more amenability to computer-based telemedicine technologies which are IP-based. Services offered by telemedicine are designed to help in improving healthcare access and information services while reducing the isolation between healthcare providers and residents in rural areas. Wireless Telemedicine reduces time and costs of patient's transportation from rural areas. Wireless Telemedicine is used in distinct areas such as consultations in neurology, cardiology, and general medicine. Telemedicine is also used for Continued Medical Education (CME), administration, research and development. Wireless Telemedicine offers so many services such as, Tele-education, Tele-consultation, Tele-monitoring, Tele-diagnosis and medical data exchange etc. In [5] wireless Telemedicine system is used to provide m-QoS. Wireless Telemedicine provides services 24 hours a day and seven days a week basis to patients in remote areas and at every where as shown in Figure 2.

\section{General Quality of Service (QoS)}

Quality of service (QoS) generally is defined as degree of satisfaction of user of the service. There are distinct QoS metrics for various network applications based on human/user factor and technology attributes. The human factor defines multiple kinds of media, for example, text, audio, video etc, and the technology attributes show distinct technological ways of using network, for example, time-dependence and symmetry. Both of these elements play important role to satisfy the desired requirement of QoS. But here we will focus only on the patient' perception for QoS. As regardless of the type of media (voice or video) there are different $\mathrm{QoS}$ requirements from the patient' point of view that determine whether the patient will accept this service or not, such as perception about video transmission delay and listening music online [16]. For more information look at Table 2.

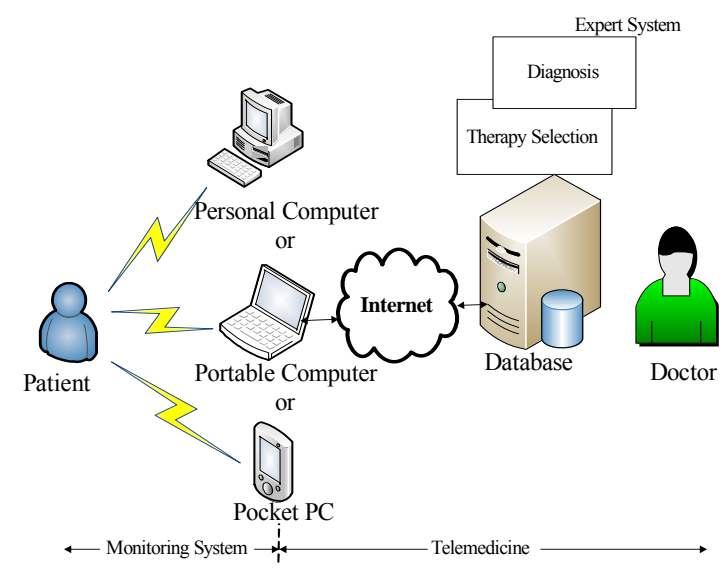

Figure 2. Architecture of wireless telemedicine system [20]. 
Table 1. Simulation results.

\begin{tabular}{|c|c|c|c|c|c|c|c|}
\hline \multicolumn{2}{|c|}{$\begin{array}{l}\text { Stream-1: Digital Subtraction } \\
\text { Angiography (DSA) }\end{array}$} & \multirow{2}{*}{$\begin{array}{c}\text { Peak_to_Mean } \\
\text { Ratio } \\
9.52\end{array}$} & \multirow{2}{*}{$\begin{array}{r}\begin{array}{r}\text { Standard } \\
\text { deviation }\end{array} \\
34.08\end{array}$} & \multirow{2}{*}{$\begin{array}{c}\begin{array}{c}\text { Delay } \\
\text { (millisecond) }\end{array} \\
9.74 \mathrm{e}-05\end{array}$} & \multirow{2}{*}{$\begin{array}{c}\begin{array}{c}\text { Jitter } \\
\text { (millisecond) }\end{array} \\
5.11 \mathrm{e}-07\end{array}$} & \multirow{2}{*}{$\begin{array}{c}\text { Average Delay } \\
\text { (millisecond) }\end{array}$} & \multirow{2}{*}{$\begin{array}{c}\begin{array}{c}\text { Average Jitter } \\
(\mathrm{ms})\end{array} \\
5.67 \mathrm{e}-08\end{array}$} \\
\hline Buffer Size & Unsmoothed & & & & & & \\
\hline КB & Smoothed & 1.94 & 0.30 & $9.51 \mathrm{e}^{-}-05$ & $5.0 \mathrm{e}-07$ & $1.056 \mathrm{e}-05$ & $5.56 \mathrm{e}-08$ \\
\hline \multirow{2}{*}{$\begin{array}{l}\text { Buffer Size } \\
=64 \mathrm{~KB}\end{array}$} & Unsmoothed & 9.52 & 34.08 & $1.94 \mathrm{e}-04$ & $1.02 \mathrm{e}-6$ & $2.15 \mathrm{e}-05$ & $1.13 \mathrm{e}-07$ \\
\hline & Smoothed & 1.77 & 0.24 & $1.92 \mathrm{e}-04$ & $1.01 \mathrm{e}-6$ & $2.13 \mathrm{e}-05$ & $1.12 \mathrm{e}-07$ \\
\hline \multirow{2}{*}{$\begin{array}{l}\text { Buffer Size } \\
=128 \mathrm{~KB}\end{array}$} & Unsmoothed & 9.52 & 34.08 & $3.89 \mathrm{e}-04$ & $2.04 e^{-}-6$ & $4.32 \mathrm{e}-05$ & $2.26 \mathrm{e}-07$ \\
\hline & Smoothed & 1.52 & 0.15 & $3.79 \mathrm{e}-04$ & $2.03 e-6$ & $4.21 \mathrm{e}-05$ & $2.25 \mathrm{e}-07$ \\
\hline \multirow{2}{*}{$\begin{array}{l}\text { Buffer Size } \\
=256 \mathrm{~KB}\end{array}$} & Unsmoothed & 9.52 & 34.08 & $7.79 \mathrm{e}-04$ & $4.09 \mathrm{e}-06$ & $8.65 \mathrm{e}-05$ & $4.54 \mathrm{e}-07$ \\
\hline & Smoothed & 1.34 & 0.061 & $7.69 \mathrm{e}-04$ & $4.08 \mathrm{e}-6$ & $8.54 \mathrm{e}-05$ & $4.53 \mathrm{e}-07$ \\
\hline \multirow{2}{*}{$\begin{array}{l}\text { Buffer Size } \\
=512 \mathrm{~KB}\end{array}$} & Unsmoothed & 9.52 & 34.08 & 0.0016 & $8.18 \mathrm{e}-06$ & $1.78 \mathrm{e}-04$ & $9.08 \mathrm{e}-07$ \\
\hline & Smoothed & 1.22 & 0.050 & 0.0015 & $8.17 \mathrm{e}-06$ & $1.67 \mathrm{e}-04$ & $9.07 \mathrm{e}-07$ \\
\hline \multirow{2}{*}{$\begin{array}{l}\text { Buffer Size } \\
=1024 \mathrm{~KB}\end{array}$} & Unsmoothed & 9.52 & 34.08 & 0.0031 & $1.63 \mathrm{e}-05$ & $3.44 \mathrm{e}-04$ & $1.81 \mathrm{e}-06$ \\
\hline & Smoothed & 1.073 & 0.049 & 0.0030 & $1.62 \mathrm{e}-05$ & $3.33 \mathrm{e}-04$ & $1.80 \mathrm{e}-06$ \\
\hline \multirow{2}{*}{$\begin{array}{l}\text { Buffer Size } \\
=2 \mathrm{MB}\end{array}$} & Unsmoothed & 9.52 & 3.25 & 0.0059 & $3.12 \mathrm{e}-05$ & $6.56 \mathrm{e}^{-}-04$ & $3.46 \mathrm{e}^{-}-06$ \\
\hline & Smoothed & 1.071 & 0.048 & 0.0058 & $3.11 \mathrm{e}-05$ & $6.44 \mathrm{e}-04$ & $3.45 \mathrm{e}-06$ \\
\hline $\begin{array}{l}\text { Buffer Size } \\
=4 \mathrm{MB}\end{array}$ & Unsmoothed & 9.52 & 3.25 & 0.0119 & $6.24 e^{-5}$ & $1.32 \mathrm{e}-03$ & $6.93 \mathrm{e}-06$ \\
\hline \multirow{2}{*}{$\begin{array}{l}\text { Buffer Size } \\
=16 \mathrm{MB}\end{array}$} & Unsmoothed & 9.52 & 4.08 & 0.0476 & $2.49 \mathrm{e}-04$ & $5.27 \mathrm{e}-03$ & $2.76 \mathrm{e}-05$ \\
\hline & Smoothed & 1.069 & 0.045 & 0.0475 & $2.48 \mathrm{e}-04$ & $5.27 \mathrm{e}-03$ & $2.75 e^{-}-05$ \\
\hline
\end{tabular}

Table 2. Requirements of general QoS and medical QoS [17-19].

\begin{tabular}{|c|c|c|c|c|c|c|c|}
\hline \multirow{2}{*}{ S. No } & \multirow{2}{*}{ Service Type } & \multicolumn{3}{|c|}{ General QoS } & \multicolumn{3}{|c|}{ Medical QoS } \\
\hline & & Data Rate (kbps) & Delay (ms) & Loss Rate & Data Rate (kbps) & Delay (ms) & Loss Rate \\
\hline 1 & Audio & $56-64 \mathrm{kbps}$ & $<150 \mathrm{~ms}$ & $<0.1 \%$ & $4-26 \mathrm{kbps}$ & $150-400 \mathrm{~ms}$ & $3 \%$ \\
\hline 2 & Video & $4-60 \mathrm{Mbps}$ & $<150 \mathrm{~ms}$ & $<0.0001 \%$ & $32-384 \mathrm{kbps}$ & $150-400 \mathrm{~ms}$ & $1 \%$ \\
\hline 3 & FTP & $11.8 \mathrm{kbps}$ & $\sim 10 \mathrm{sec}$ & 0 (zero) & $16.99 \mathrm{kbps}$ & $177.6 \mathrm{~ms}$ & 0 (zero) \\
\hline 4 & VOIP & $64 \mathrm{kbps}$ & $\begin{array}{c}<150 \mathrm{~ms} \text {, phone to } \\
\text { phone delay }\end{array}$ & $<1 \%$ & $500 \mathrm{kbps}$ & $\begin{array}{c}150-240 \mathrm{~ms} \\
\text { (downlink) } \\
200 \mathrm{~ms} \text { (uplink) }\end{array}$ & $0.01 \%$ \\
\hline 5 & Video conferencing & $24-1920 \mathrm{kbps}$ & $<150 \mathrm{~ms}$ & $<0.01 \%$ & $640 \mathrm{kbps}-5 \mathrm{Mbps}$ & $<250 \mathrm{~ms} \mathrm{E} 2 \mathrm{E}$ & $1 \%$ \\
\hline 6 & Images & $<100 \mathrm{kbps}$ & $<10 \mathrm{sec}$ & NA & $<1 \mathrm{Mbps}$ & $\sim 10 \mathrm{sec}$ & NA \\
\hline 7 & Web browsing & $\sim 10 \mathrm{kbps}$ & $<4$ sec., per page & 0 (zero) & $10 \mathrm{kbps}$ & $\sim 2$ sec. & 0 (zero) \\
\hline 8 & Email & $<10 \mathrm{kbps}$ & $<4$ sec. & 0 (zero) & $<30.5 \mathrm{kbps}$ & $<400 \mathrm{~ms}$ & 0 (zero) \\
\hline
\end{tabular}




\section{Medical Quality of Service (m-QoS)}

Medical QoS (m-QoS) can be defined as the augmented requirements of critical mobile healthcare applications with respect to the traditional wireless QoS requirements. The main m-QoS metrics in video streaming environment are the throughput, packet loss rate; end to end delay and jitter [17]. The m-QoS in the network ensures that patient gets usable services. Every medical service has different requirement which depends on the type of the traffic, type of the service and the context in which service is invoked. Medical applications generate traffic with diverse network metrics which plays vital role to optimize m-QoS. Here, statistical similarity between the smoothed and unsmoothed medical video transmission is performed using different network metrics. Medical QoS is the ability to provide different priority to different applications, patients, or data flows to guarantee certain level of performance for medical applications such as, Tele-monitoring. m-QoS metrics need to be guaranteed by delivering satisfactory wireless multimedia services. There are primarily three ways in which $\mathrm{m}-\mathrm{QoS}$ is provided. First, Inter frame Space (IFS) or different contention window, second, weighted fair queuing (WFQ) and third, Admission control mechanism. m-QoS is optimized with optimal smoothing algorithm in terms of high peak data rate, peak-to-mean ratio, and standard deviation, delay and jitter values at different client buffer size as shown in Table 1. The clear difference between mQoS and general QoS is shown in Table 2.

\section{Difference between General and Medical Video}

Recently, video is the convenient and effective source of conveying any kind of information. All video formats achieve the same basic goal, for example, they store black and white or colored information as electronic lines that make up a video frames recorded per second and varies, depending on the video standard, the format supports, for example, NTSC formats are recorded at 29.9 fps and PAL at $25 \mathrm{fps}$. Both general and medical videos are characterized by the same parameters such as, encoding method, frame rate, sampling rate, video standard supported, aspect ratio of frames, aspect ratio of pixels, sampling rate and bandwidth etc plays important role to analyze any video as shown in Table 3.

\section{Optimal Smoothing of Offline Medical Video}

\subsection{Problem Statement}

In particular, supporting robust and smooth medical video transmission over wireless networks is a challenging problem primarily due to three factors: fixed (low)
Table 3. Difference between general video \& medical video $[17,18]$.

\begin{tabular}{|c|c|c|c|}
\hline S. No & Parameter & General Video & Medical Video \\
\hline 1 & Video Encoder & MPEG-1, MPEG-4 & M-JPEG, H.264 \\
\hline 2 & Frame Rate & $\begin{array}{l}15 \mathrm{fps}, 25 \mathrm{fps} \\
\text { PAL/SECAN }\end{array}$ & 30 fps NTSC \\
\hline 3 & Video standard & PAL & NTSC \\
\hline 4 & $\begin{array}{l}\text { Aspect ratio of } \\
\text { frames }\end{array}$ & $(3: 2,4: 3,5: 4)$ & 16:9 (HDTV) \\
\hline 5 & $\begin{array}{l}\text { Aspect ratio of } \\
\text { pixels }\end{array}$ & {$[704 \times 576=12: 11]$} & {$[704 \times 480=10: 11]$} \\
\hline 6 & Color mapping & $4: 2: 2$ & $4: 4: 4$ \\
\hline 7 & Sample rate & $96 \mathrm{KHz}$ & $44.1 \mathrm{KHz}$ \\
\hline 8 & Bandwidth & $100-500 \mathrm{MB}$ & $\sim 1 \mathrm{~GB}$ \\
\hline
\end{tabular}

allocated and variable demanding bandwidth, varying nature of transmission channel and end-to-end QoS and delay guarantee. So, to resolve these problems we used optimal smoothing algorithm.

\subsection{Optimal Smoothing Algorithm}

In formulating optimal smoothing algorithm, we consider discrete time model at the frame level. That is, $t \in\{1,2, \cdots, N\}$, where $N$ is the number of frames in the video.

Algorithm Parameters:

$N$ : Number of frames in the video.

$P$ : Start-up delay in frame periods (time slots).

$B$ : Client buffer capacity to store unplayed video frames.

$L(t)$ : Size of the frame in time slot $t$ in bits, $t=1,2, \cdots, N$.

$D(t)$ : Cumulative amount of data (in bits) consumed by client.

$a(t)$ : Amounts of data (in bits) sent by the server at time, $t=1,2, \cdots, N$.

$A(t)$ : Cumulative amount of data sent by server at $[1, t]: \sum_{i=1}^{t} a(i)$.

$B(t)$ : Maximum cumulative data received by the client over $[1, t]$, without buffer overflow.

Let $a(t)$ be the amount of data sent by the server at time $t$. We assume data are consumed from buffer after any arrival at time $t$. When the client has fixed buffer size $B$, we have $B(t)=\min \{D(t-1)+B, D(N)\}$ for $t=2, \cdots, N$, with $B(1)=B$ and $B(0)=0$. It is not necessary for $B(t)$ to lie at fixed offset above $D(t)$. The $N$ dimensional real vector $S=[a(1), \cdots, a(N)]$ is said to represent a feasible server transmission schedule 
over $[1, N]$ if the client buffer neither starves nor overflows during stream playback, i.e., if $D(t) \leq A(t) \leq B(t)$ for all $t$, as shown in Figure 3 which represent variation in the amount of data in the client buffer over multiple time slots. The difference between $B(t)$ and $D(t)$ is the client buffer size. The cumulative data $A(t)$ transmitted must be more than $D(t)$ but less than $B(t)$ to ensure that the client buffer neither overflows nor starves. In every time slot, we adjusted to minimize high peak data rate. If the start up delay $P \geq 1$ time slots, the cumulative data transmitted by the end of the $P_{\text {th }}$ time slot $A(P)$, should be larger than the size of the first frame $L(1)$, but smaller than the client buffer size, i.e., $L(1) \leq A(P) \leq B$. In this range, we can choose the optimal $A(P)$ so as to minimize high peak data rate and reduce variability. By the end of the $(P+1)_{\text {th }}$ time slot, transmitted data $A(P)+a(P+1)$ is more than the sum of the first two frames, but smaller than the sum of the buffer size and first frame, i.e.,

$L(1)+L(2) \leq A(P)+a(P+1) \leq B+L(1)$. After solving this inequality, $A(P)$ is constant and we can adjust $a(P+1)$ for minimum peak data rate and lowest rate variability. More generally, at the end of the $(P+k)_{t h}$ time slot, $A(P), a(P+1), a(P+2), \cdots, a(P+k-1)$ are constants and $a(P+k)$ is adjusted to reduce rate variability and high peak data rate. That is,

$$
\sum_{i=1}^{k+1} L(i) \leq A(P+k-1)+a(P+k) \leq B+\sum_{i=1}^{k} L(i)
$$

In Equation (1) $a(P+k)=0$ can satisfy the inequality $A(P+k-1)-\sum_{i=1}^{k+1} L(i) \geq$ temp, where temp is constant.

As temp $=0$ satisfy the inequality to avoid unexpected frame loss. We can set temp equal to the sum of the several frame sizes to have a safety buffer of prefetched frames that allows continuous playback during wireless outages.

Let $C_{\max }$ be the maximum rate at which server trans-

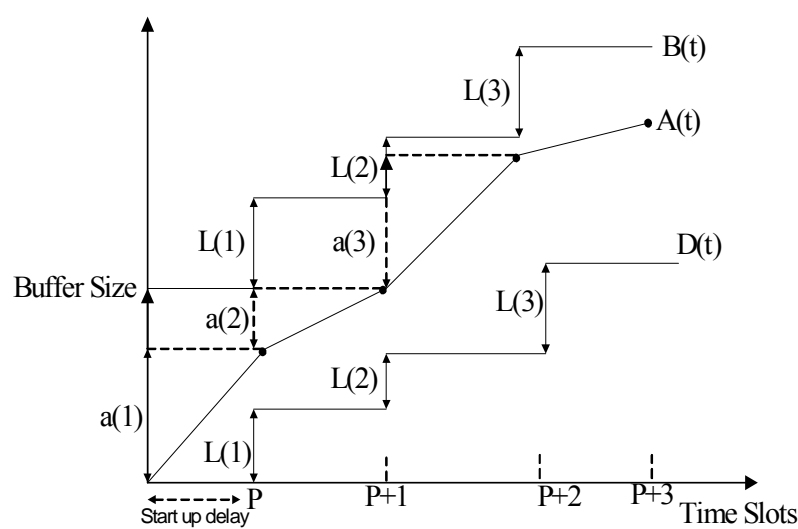

Figure 3. Diagram of video data received and consumed by client. mits over a given interval $[a, b]$, without overflowing the client buffer, starting from initial buffer level $q$, as shown in Equation (2)

$$
C_{\max }=\min _{a+1 \leq t \leq b} \frac{B(t)-(D(a)+q)}{t-a}
$$

and $t_{B}$ is the latest time $t$ at which client buffer is full when the server transmits at $C_{\max }$ over $[a, b]$, starting with initial buffer level $q$.

$$
t_{B}=\max _{a+1 \leq t \leq b}\left\{t: \frac{B(t)-(D(a)+q)}{t-a}=C \max \right\}
$$

In the same way, $C_{\min }$ is the minimum rate at which server transmits over a given interval $[a, b]$ such that the client buffer never starves, starting from initial client buffer level $q$.

$$
C_{\min }=\max _{a+1 \leq t \leq b} \frac{D(t)-(D(a)+q)}{t-a}
$$

and $t_{D}$ is the latest time at which the client buffer is empty when the server transmits at $C_{\min }$ over $[a, b]$, starting with the initial buffer level $q$.

$$
t_{D}=\max _{a+1 \leq t \leq b}\left\{t: \frac{D(t)-(D(a)+q)}{t-a}=C \min \right\}
$$

video transmission over an interval $[a, b]$ will be feasible when $C_{\max } \geq C_{\min }$ while computing over $[a, b]$ as shown in Figure 3.

\section{Simulation Results}

Here we have compared the unsmoothed video transmission with the smoothed video transmission, on frame by frame basis with respect to bit rate peak-to-mean ratio, standard deviation of bit rate, delay, average delay, jitter and average Jitter. Transmission scheme is simulated with 30-minute VBR MPEG-4 QCIF format encodings from the movie Digital Subtraction Angiography (DSA). The video stream with range of bit rates is available at $\mathrm{http}: / /$ traces.eas.asu.edu and their values are shown in Table 4. In Table 1 we compared the performance of stream-1 for nine different client buffer size values at start-up delay of $2 \mathrm{sec}$. if we slightly increase the start-up delay value than peak-to-mean ratio, standard deviation reduces but delay and jitter values increase at small extent. If we increase the buffer size then peak-to-mean ratio and standard deviation reduces as shown in Table 1 and Figure 4. In Table 1 we analyzed tradeoff between different client buffer sizes and network metrics using optimal smoothing algorithm to optimize m-QoS. It is cleared that when buffer size increases then peak-tomean ratio and Std. dev reduces (i.e. becomes smooth) while delay and jitter value gets larger. 


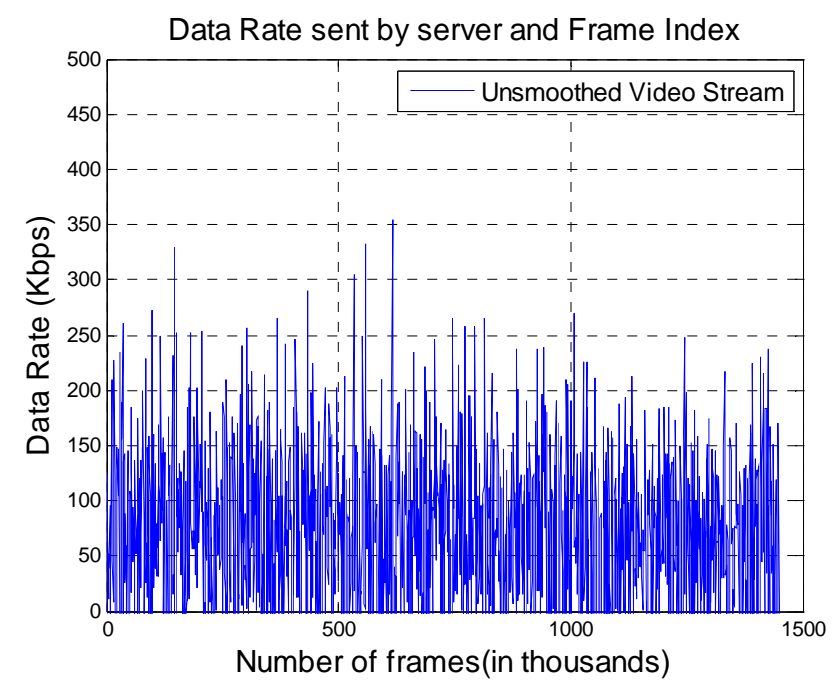

(a)

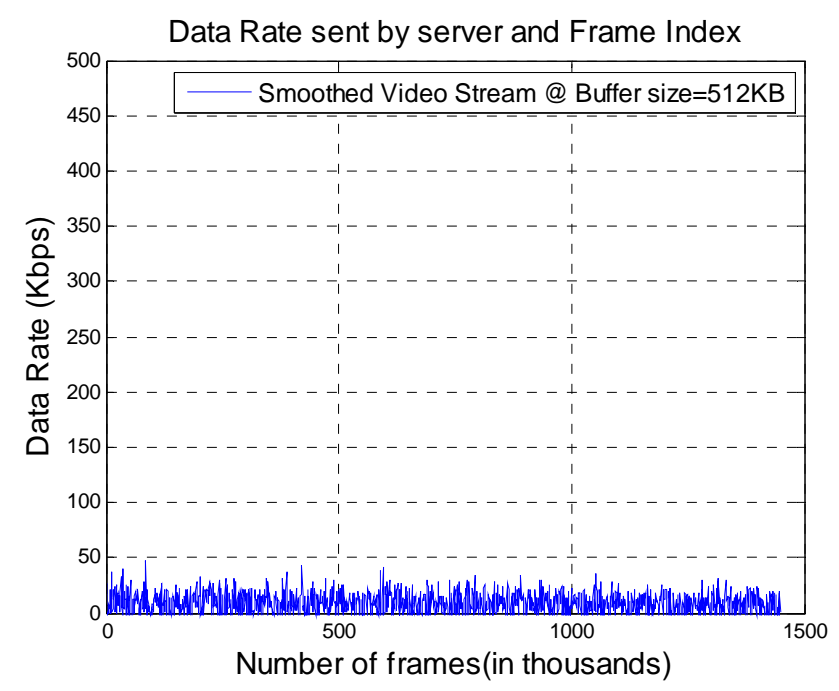

(c)

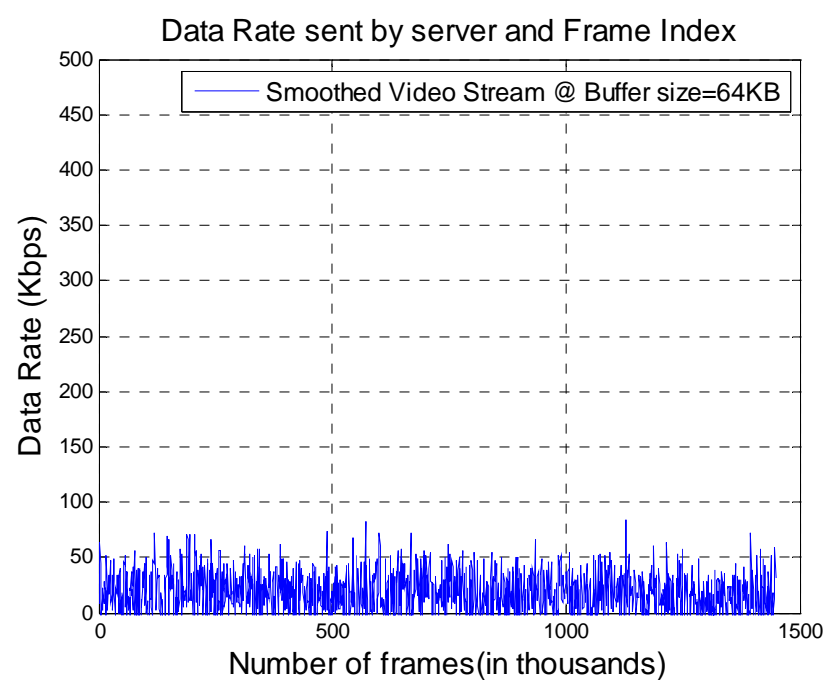

(b)

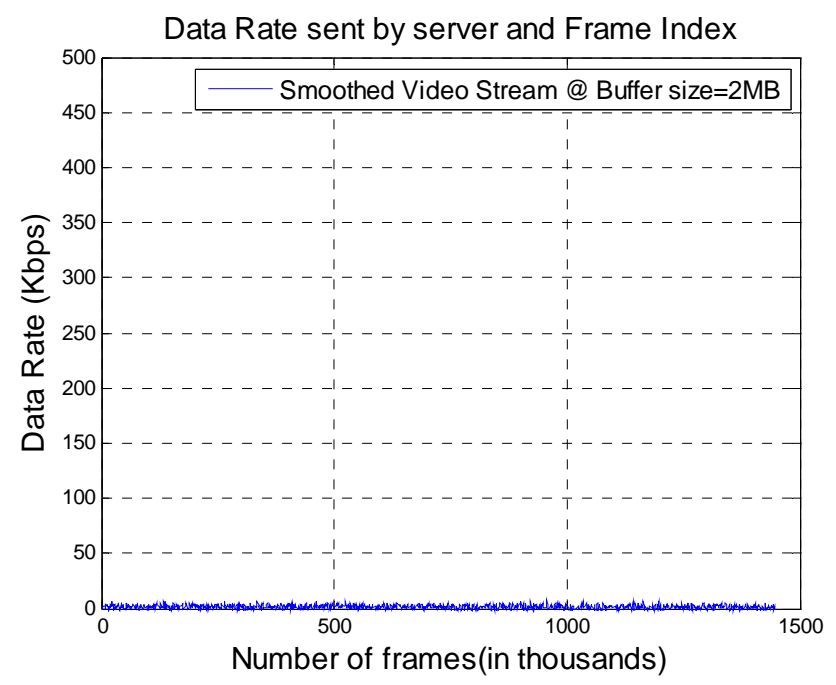

(d)

Figure 4. Offline optimal smoothing algorithm. (a) Unsmoothed video stream; (b) Smoothed video stream at buffer size of 64 KB; (c) Smoothed video stream at buffer size of 512 KB, (d) Smoothed video stream at buffer size of 2 MB, X-axis shows frame index in thousands and $\mathrm{Y}$-axis represents data rate in Kbps sent by server.

\section{Our Improvement in Results}

After deep analysis of different metrics of general video, general QoS, medical video and medical QoS requirements we come to the main point and compared standard values of network metrics with values obtained from our simulation results, we found that value of delay, jitter obtained from our experiment are in microseconds as shown in Table 1 while standard value of same metrics are in milliseconds as shown in Tables 5 and $\mathbf{6}$, so there is much more difference between standard and observed values of different parameters, also data rate values of peak-to-mean ratio and standard deviation metrics are very small and optimum from our experiment than the standard values. In other words we can say that results of these metrics obtained from our experiment are suitable for the requirements of medical video and medical QoS, while standard values of these metrics are very large and require very expensive tools and complex techniques to achieve. We used very simple and an efficient technique named Optimal Smoothing Algorithm to satisfy the requirements of medical video and medical-QoS. In all aspects optimal smoothing algorithm performs better.

\section{Conclusion and Future Work}

We applied optimal smoothing algorithm to optimize m-QoS, established trade-off between client buffer size, peak-to-mean ratio, standard deviation, delay, jitter, average delay and average jitter. Also we compared general QoS with medical QoS and general video with medical video in terms of standard values of parameters and 
Table 4. Simulation parameters.

\begin{tabular}{cc}
\hline $\begin{array}{c}\text { Stream-1 Digital Subtraction } \\
\text { Angiography (DSA) }\end{array}$ & I-P-B Quant scale, 30-030-30 \\
\hline Encoding standard & MPEG-4, QCIF (176X144) \\
Peak-to-Mean Ratio & 9.864 \\
Mean_bit_rate (Mbps) & 0.901 \\
Peak_bit_rate (Mbps) & 8.89 \\
1-GOP (Group Of Pictures) & 12 frames \\
Time of one frame & $33 \mathrm{~ms}$ \\
Total time of video & 30 mints $=30 \times 60=1800 \mathrm{sec}$. \\
Total number of frames & 54000 \\
Start_up_delay of P frames & $2 \mathrm{sec}$. \\
Bit_rate (kbps) & $2.691 \mathrm{e}+07$ \\
Average_bit_rate & $2.82 \mathrm{e}+05$ \\
(kbps)-unsmoothed video & \\
Average_bit_rate $(\mathrm{kbps})$-smoothed & video \\
Number of clients, C & $2.88 \mathrm{e}+05$ \\
Total_bits & 93242776 \\
\hline
\end{tabular}

Table 5. General video requirements [17].

\begin{tabular}{ccccc}
\hline S. No & $\begin{array}{c}\text { Application/Service } \\
\text { Type }\end{array}$ & $\begin{array}{c}\text { Delay } \\
(\mathrm{ms})\end{array}$ & $\begin{array}{c}\text { Jitter } \\
(\mathrm{ms})\end{array}$ & $\begin{array}{c}\text { Data Rate } \\
(\mathrm{bps})\end{array}$ \\
\hline 1 & Video broadcasting & $<150$ & $<100$ & $15-34 \mathrm{M}$ \\
2 & Interactive VoD & $<150$ & $<150$ & $\begin{array}{c}28.8-500 \mathrm{~K}, \\
\text { single video }\end{array}$ \\
3 & Videoconference & $<150$ & $<400$ & $<64 \mathrm{~K}$ \\
4 & Videophony & $<100$ & $<400$ & $<64 \mathrm{~K}$ \\
5 & VoIP & $<100$ & $<400$ & $8 \mathrm{~K} @$ G.729
\end{tabular}

Table 6. Medical video requirements [18].

\begin{tabular}{ccccc}
\hline S. No & $\begin{array}{c}\text { Application/Service } \\
\text { Type }\end{array}$ & $\begin{array}{c}\text { Delay } \\
(\mathrm{ms})\end{array}$ & $\begin{array}{c}\text { Jitter } \\
(\mathrm{ms})\end{array}$ & $\begin{array}{c}\text { Data Rate } \\
(\mathrm{bps})\end{array}$ \\
\hline 1 & Video broadcasting & $150-400$ & 30 & $32-384 \mathrm{~K}$ \\
2 & Interactive VoD & 150 & 20 & $4-6 \mathrm{M}$ \\
3 & Videoconference & 200 & 20 & $64 / 128 \mathrm{~K}$ \\
4 & Videophony & 100 & 10 & $128 \mathrm{~K}$ to $10 \mathrm{M}$ \\
5 & VoIP & $\begin{array}{c}150 \text { (downlink) } \\
\text { 200 (uplink) }\end{array}$ & 20 & $127 \mathrm{~K}$ \\
\hline
\end{tabular}

values from our experiments. We observed that our results are optimal than the standard values of parameters. Our simulation results show that $\mathrm{m}-\mathrm{QoS}$ is optimized by reducing rate variability, high peak data rate and network metrics. We proved experimentally that optimal smoothing algorithm fulfills requirement of $\mathrm{m}-\mathrm{Q} o \mathrm{~S}$. In near future, we will develop online smoothing algorithm then test and verify both offline and online algorithms on hardware for offline and live medical applications respectively.

\section{Acknowledgements}

This work was supported in part by the National Natural Science Foudation of China (Grant No. 81101127), the National S\&T Major Project of China (Grant No. 2011ZX03005-001), the Guangdong S\&T Major Project (Grant No. 2011A090100025), and the Shenzhen Basic Research Project (Grant No. JC201005270306A)

\section{REFERENCES}

[1] P. Malindi, "QoS in Telemedicine," Walter Sisulu University, Mthatha, 2011.

[2] P. Malindi and M. T. Khan, "Provisioning QoS for IP-Based Rural Telemedicine System," Proceedings of 3rd International Conference on Broadband Communications, Information Technology and Biomedical Applications, Pretoria, November 2008, pp. 499-504.

[3] J. D. Salehi, Z.-L. Zhang, J. Kurso and D. Towsley, "Supporting Stored Video: Reducing Rate Variability and End-to-End Resource Requirements through Optimal Smoothing," IEEE/ACM Transaction on Networking, Vol. 6, No. 4, 1998, pp. 397-410.

[4] Y. Li, M. Reisslein and C. Chakrabarti, "Energy Efficient Video Transmission over Wireless Link," IEEE Transaction on Vehicular Technology, Vol. 58, No. 3, 2009, pp. 1229-1244.

[5] A. Alinejad, N. Philip and R. S. H. Istepanian, "Mapping of Multiple Parameters M-Health Scenario to Mobile WiMAX QoS Variables," 33rd Annual International Conference of IEEE EMBS, Boston, 30 August-3 September 2011, pp. 1532-1535.

[6] Y. Mansour, B. Patt-Shamir and O. Lapid, "Optimal Smoothing Schedules for Real-Time Streams," January 2000.

[7] P. Malindi and M. T. Khan, "Provisioning QoS for IPBased Rural Telemedicine System," Proceedings of 3rd International Conference on Broadband Communications, Information Technology and Biomedical Applications, Pretoria, November 2008, pp. 499-504.

[8] R. S. H. Istepanian, N. Y. Philip and M. G. Martini, "Medical QoS Provision Based on Reinforcement Learning in Ultrasound Streaming over 3.5 G Wireless Systems," IEEE Journal on Selected Areas in Communication, Vol. 27, No. 4, 2009, pp. 566-674.

[9] W. Chi, "Performance Evaluation of Smoothing Algo- 
rithm for Prerecorded VBR Video," 1999.

[10] Taceittin, "Smoothing Algorithm for Delivery of Compressed Video," 1999.

[11] R.-I. Chang, "Designing ON-OFF CBR Transmission for Jitter Free VBR Media in Real Time Networks,” 1997.

[12] T. H. Szymanski and D. Gilbert, "Provisioning MissionCritical Telerobotic Control System over Internet Backbone Networks with Essential-Perfect QoS," IEEE Journal on Selected Areas in the Communication, Vol. 28, No. 5, 2010, pp. 630-643.

[13] B. Woodward and R. S. H. Istepanian, "Design of Telemedicine System Using a Mobile Telephone," IEEE Transaction on Information Technology in Biomedicine, Vol. 5, No. 1, 2001, pp. 13-15. doi:10.1109/4233.908361

[14] J. Rexford and Towsely, "Smoothing VBR Video in an Internet," IEEE/ACM Transaction on Networking, Vol. 7, No. 2, 1999.

[15] S. Sen, J. L. Rexford and J. K. Dey, “Online Smoothing of VBR Streaming Video," IEEE Transaction on Multi- media, Vol. 2, No. 1, 2000, pp. 37-48. doi: $10.1109 / 6046.825793$

[16] Z. L. Wang, "A Fast Algorithm of Optimal Smoothing Scheme for Stored Video," 2009.

[17] Y. Chen, T. Farley and N. Ye, "QoS Requirements of Network Applications on the Internet," Information Knowledge Systems Management, Vol. 4, No. 1, 2004, pp. 55-76.

[18] L. Skorin-Kapov, et al., "Analysis of QoS Requirements for E-Health Services and Mapping to Evolved Packet System QoS Classes," International Journal of Telemedicine and Applications, Vol. 2010, 2010, 18 p. doi: $10.1155 / 2010 / 628086$

[19] D. Niyato, et al., "IEEE 802.16/WIMAX-Based Broadband Wireless access and Its Applications for Telemedicine/E-Health Services," IEEE Wireless Communications, Vol. 14, No. 1, 2007, pp. 72-83.

[20] http:// www.google.com/telemedicine/images 